\title{
Atlas Strips Upgrade
}

\section{Mercedes Miñano ${ }^{1}$ on behalf of the ATLAS SCT Collaboration}

Instituto de Física Corpuscular (IFIC)

Edificio Institutos de Investigación

Apartado de Correos 22085

E-46071 Valencia - Spain

E-mail: Mercedes.Minanodific.uv.es

The LHC proton-proton collider is just starting to run at CERN. The luminosity of the LHC will increase over several years of operation reaching $10^{34} \mathrm{~cm}^{-2} \mathrm{~s}^{-1}$. Over the last years an upgrade of the LHC, the SuperLHC (SLHC), towards higher luminosities has been discussed as an extension of the LHC physics program. This increase in luminosity is foreseen around 2020 and will require challenging improvements in the detectors. In particular, the ATLAS experiment will require a new tracking system capable of dealing with an instantaneous luminosity of $10^{35} \mathrm{~cm}^{-2} \mathrm{~s}^{-1}$, one order of magnitude higher radiation levels and between 5 and 10 years of further operation. Many R\&D activities for the SLHC are now starting and the corresponding collaboration frameworks with worldwide partners are being stablished. One of this $\mathrm{R} \& \mathrm{D}$ programs is devoted to the development of radiation hard silicon sensors to operate to the expected SLHC doses involving many particle physics groups and several leading manufacturers of silicon sensors. In this framework, new silicon sensors (like ptype silicon) and new technologies (like 3D devices) are investigated. In parallel, alternative module concepts have been developed with higher levels of integration. We will report on the status of the R\&D projects on radiation hard silicon strip detectors for the ATLAS Tracker upgrade.

VERTEX 2009 (18 ${ }^{\text {th }}$ workshop) - VERTEX 2009

Veluwe, the Netherlands

September 13-18, 2009

\footnotetext{
$1 \quad$ Speaker
} 


\section{Introduction}

The first beams are circulating in the Large Hadron Collider (LHC) at CERN. It is expected to reach its designed peak luminosity $\left(10^{34} \mathrm{~cm}^{-2} \mathrm{~s}^{-1}\right)$ in a few years and deliver to the experiments an integrated luminosity of $300 \mathrm{fb}^{-1}$ in the first five years of operation.

An upgrade of the accelerator has been considered in terms of its luminosity. The upgrade program comprises two phases. Phase I consists of the replacement of the inner triplet focusing magnets with larger aperture ones, and bringing on-line a new linac (Linac-4) [1]. Phase I should allow an increase of luminosity up to $3 \times 10^{34} \mathrm{~cm}^{-2} \mathrm{~s}^{-1}$. Phase II should be implemented once the Phase I reaches the end of the magnet lifetime $\left(700 \mathrm{fb}^{-1}\right)$. The second phase will bring in more improvements in the injector chain and it is expected in 2020; various options are under study to find the best way to increase luminosity to 10 times nominal. Coping with the very high data rates and radiation levels will require major changes to the ATLAS detector. In particular, the inner tracking system will have to be replaced before proceeding to the SLHC luminosity target of $10^{35} \mathrm{~cm}^{-2} \mathrm{~s}^{-1}$ [2]. Once the SLHC luminosity is reached, in five years of operation an integrated luminosity of about $3000 \mathrm{fb}^{-1}$ will be accumulated.

The two main implications of this very high luminosity regarding detector performance are a pile-up of about 400 proton-proton interactions per collision and a large increase of particle flow. These issues are of particular importance for the tracking detectors located close to the LHC interaction region, where the highest radiation doses occur. Therefore, the ATLAS experiment needs to replace the entire inner tracking [3]. An increase of the pile-up implies finer granularity for the new tracking detectors to keep the occupancy acceptably low. In order to cope with the increase of the particle fluence, a detector with enhanced radiation hardness should be designed for the tracking system.

\subsection{ATLAS Semiconductor Tracker Upgrade}

The current ATLAS Inner Tracker consists of a Silicon Pixel Detector (Pixel), a Silicon Strip Detector (SCT) and a Transition Radiation Tracker (TRT) [4]. The complete Inner Tracker will be fully replaced at the upgrade phase II, since the current one was designed for $740 \mathrm{pb}^{-1}$. The Transition Radiation Tracker detector system of ATLAS is inadequate for running at the expected luminosities; an all silicon tracker should be implemented. The replacement tracker would need completely new designs for the factor 10 higher radiation doses and would have much greater granularity to cope with the much higher occupancies. In the new design, the SCT will be extended from 4 silicon strip layers within $30-51 \mathrm{~cm}$ to 5 layers at radii in $38-95 \mathrm{~cm}$ and with 5 double-sided disks in each end-cap. The three outer layers will replace the actual TRT. For the inner most region a new Pixel detector has to be designed.

The inner three layers are designed to contain short $24 \mathrm{~mm}$-long strips (short strip layers), while the outer two layers will be containing $96 \mathrm{~mm}$-long strip detectors (long strip layers). The 
pitch for long and short strips is expected to be about $75 \mu \mathrm{m}$. The design is expected to keep the occupancy of $1.6 \%$ at the innermost radius, which is considered adequate.

Concerning radiation damage, the classic concept of p-on-n silicon microstrip detectors as used in the current SCT needs to be abandoned for the SLHC. These p-on-n detectors have been proven to be adequate for the radiation levels of the present LHC, and would be quickly rendered useless by the large SLHC radiation fluence. The short strip detectors are required to withstand $9 \times 10^{14} 1 \mathrm{MeV}$ equivalent neutron $\left(\mathrm{n}_{\mathrm{eq}}\right) \mathrm{cm}^{-2}$ consisting about $50 \%$ of neutrons and about $50 \%$ of charged particles. The outer detectors will have to cope with fluences up to $4 \times 10^{14}$ $\mathrm{n}_{\mathrm{eq}} \mathrm{cm}^{-2}$ consisting mostly of neutrons. The pixel region will consist of 4 silicon layers withstanding a radiation dose of $10^{16} \mathrm{n}_{\mathrm{eq}} \mathrm{cm}^{-2}$ at the innermost layer. There are several alternatives on sensor R\&D on going: 3D, Diamond or planar (n-on-n) [5].

Consequently, a massive R\&D program is underway to develop silicon sensors with sufficient radiation hardness to operate at the SLHC [6].

\section{Radiation Hard Technologies}

P-type microstrip detectors (n-type strips processed into p-type bulk) are being investigated for the ATLAS Inner Tracker upgrade. A batch of test sensors, called ATLAS07 [7], has been fabricated by Hamamatsu Photonics [8] on high resistivity float zone silicon substrate. On each $15 \mathrm{~cm}$-diameter wafer there are 24 miniature strip detectors $\left(1 \mathrm{x} 1 \mathrm{~cm}^{2}\right)$ and a full size prototype sensor $\left(9.75 \times 9.75 \mathrm{~cm}^{2}\right)$. The sensors are $320 \mu \mathrm{m}$ thick. They have AC coupled readout, $\mathrm{n}$ strips biased through polysilicon bias resistors connected to a bias ring and a single guard ring.

The miniature sensors have 104 strips with a pitch of $74.5 \mu \mathrm{m}$. The full size prototype sensors have $1280(\mathrm{x} 4)$ strips. They are formed by 4 segments of $2.4 \mathrm{~cm}$ strip length. Two of the four segments are designed with "axial" strips where the strips are parallel to the sensor edges, and two with "stereo" strips where the strips are inclined (40 mrad) to the sensor edges. The nstrip isolation method is summarized in Table 1.

Table 1: N-strip isolation method and surface concentration (ions $\left./ \mathrm{cm}^{2}\right)$.

\begin{tabular}{ccc}
\hline & p-Stop & p-Spray \\
\hline ATLAS 07 & $2 \times 10^{12}, 4 \times 10^{12}, 1 \times 10^{13}$ & $2 \times 10^{12}$ \\
\hline
\end{tabular}

\subsection{ATLASO7 under proton and neutron irradiation}

ATLAS miniature sensors have been used for irradiation studies. Several institutes [9] of the ATLAS Upgrade Silicon Detector R\&D collaboration were involved in the measurements. A set of samples was irradiated with $70 \mathrm{MeV}$ protons to fluences of $2.3,6$, and $13 \times 10^{14} \mathrm{n}_{\mathrm{eq}} \mathrm{cm}^{-2}$ at CYRIC [10]. Another set was irradiated with neutrons at the TRIGA Nuclear Reactor to fluences of 2, 5, and $10 \times 10^{14} \mathrm{n}_{\mathrm{eq}} \mathrm{cm}^{-2}$ [11]. Charge collection in the sensors was evaluated using a ${ }^{90} \mathrm{Sr}$ source and infrared laser rays. Previously, non-irradiated sensors were characterized to ensure the agreement between the measuring systems done at the different sites. Fig. 1 shows a good agreement in the measured charge collection performance in the different laboratories for non-irradiated detectors. All measurements are consistent within the systematic errors. 


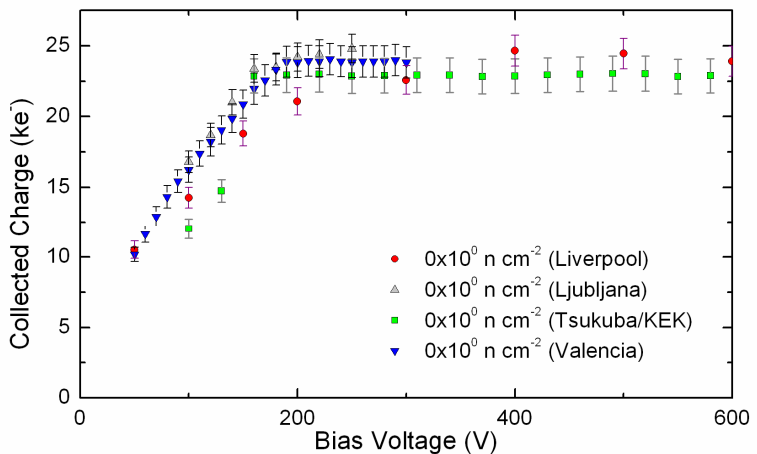

Fig. 1. Collected charge as a function of the bias voltage for non-irradiated sensors measured at different sites.

The collected charges after neutron and proton irradiation are shown in Fig. 2 and Fig. 3 respectively for the expected fluences at the short strip layers (a) and the long strip layers (b). The samples from Ljubljana and Tsukuba were previously annealed therefore the charge collection improves by about $20 \%$. The radiation damage seems more severe for neutrons than for protons for the same fluences scaled by the NIEL factor [12]. A full compilation of all these collected charge measurements is available at [13].

(a)

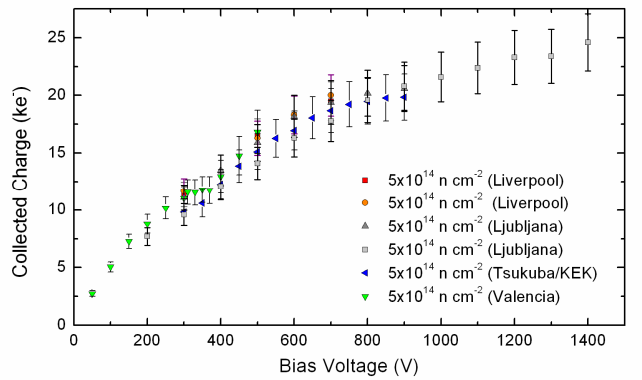

(b)

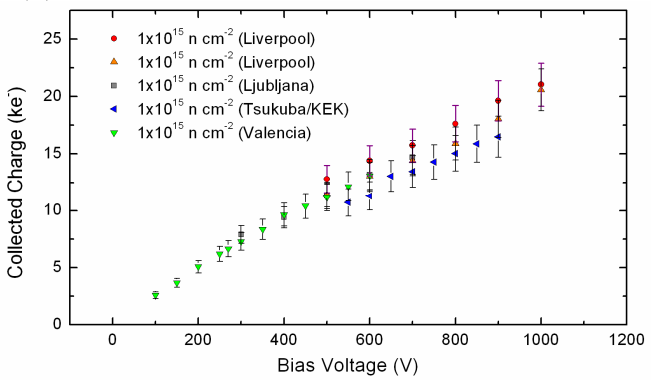

Fig. 2. Collected charge vs. bias voltage for (a) sensors irradiated with neutrons at $5 \times 10^{14} \mathrm{n}_{\mathrm{eq}} \mathrm{cm}^{-2}$ and (b) at $10 \times 10^{14} \mathrm{n}_{\mathrm{eq}} \mathrm{cm}^{-2}$.

(a)

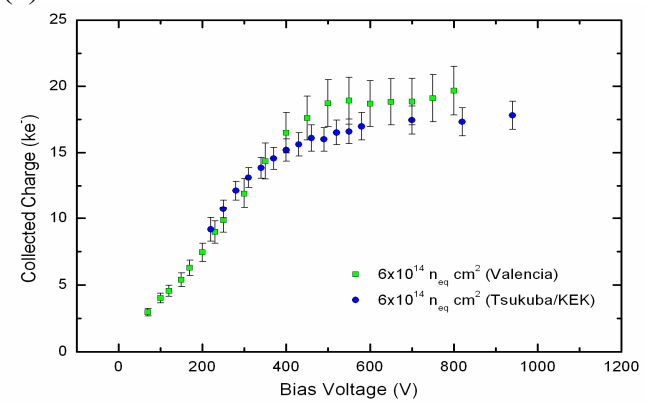

(b)

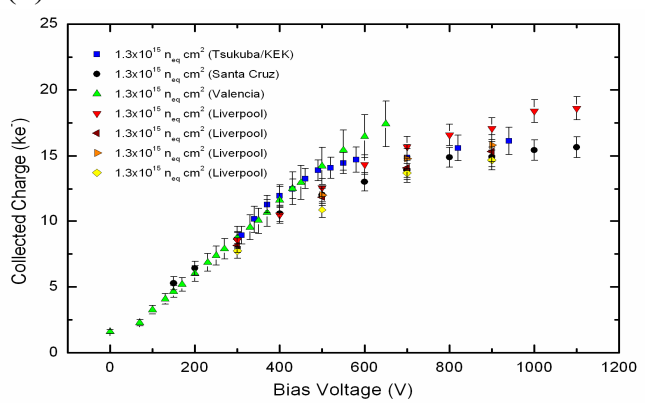

Fig. 3. Collected charge vs. bias voltage for (a) sensors irradiated with $70 \mathrm{MeV}$ protons at $6 \times 10^{14} \mathrm{n}_{\mathrm{eq}} \mathrm{cm}^{-2}$ and (b) $13 \times 10^{14} \mathrm{n}_{\mathrm{eq}} \mathrm{cm}^{-2}$.

The collected charge at $500 \mathrm{~V}$ is estimated to be $14-16 \mathrm{k}$ electrons for neutron irradiation and 16-19k electrons for proton irradiation at the expected doses in the long strip layers. For an expected noise of 950 electrons (calculated from capacitance 
associated to the strip length) one can achieve a signal/noise ratio $(\mathrm{S} / \mathrm{N})$ of approximately 16 (18) for sensors irradiated with neutrons (protons). In the case of short strips at $500 \mathrm{~V}$, the estimated collected charge is $11-13 \mathrm{k}$ electrons for neutrons and 12$14 \mathrm{k}$ electrons for protons reaching a $\mathrm{S} / \mathrm{N}$ of 20 in both cases, considering an input noise of 600 electrons. These $\mathrm{S} / \mathrm{N}$ values are feasible for detector operation.

The full depletion voltage (FDV) as a function of the fluence has also been studied (Fig.4). As observed, detectors irradiated with neutrons require a higher FDV than those irradiated with protons. The full depletion for the neutron samples could be reached at $800 \mathrm{~V}$ for the doses expected in the long strip layers. In these layers the neutron contribution to the radiation damage will be higher than the proton contribution. For the doses expected at the short strip layer, the full depletion will be reached beyond $1000 \mathrm{~V}$ when neutron irradiation is considered. However, at these radii the proton contribution is as important as the neutron one and this mixed irradiation would decrease the FDV [14].

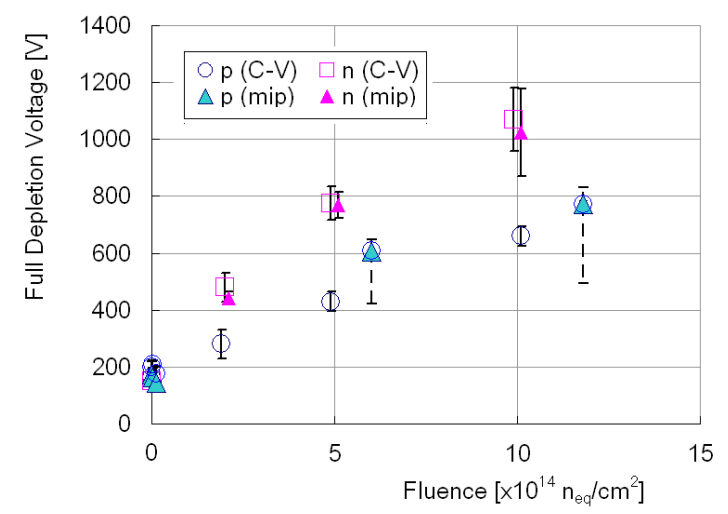

Fig. 4. Full depletion voltage as function of the fluence, measured with two different methods: CCE-V (mip) and Capacitance-V curves. Figures in blue for protons and in pink for neutrons [15].

\subsection{ATLAS07 full size prototype sensors}

The ATLAS07 full size sensors were characterized in terms of electrical parameters (leakage current, depletion voltage, coupling capacitance and bias resistance). Several institutes of the ATLAS Upgrade Silicon Detector R\&D collaboration [16] were involved in the measurements.

The leakage current level for all the sensors was $200-350 \mathrm{nA}$ at $600 \mathrm{~V}$ while the ATLAS Technical Specifications [17] require a leakage current below $200 \mu \mathrm{A}$ at $600 \mathrm{~V}$. The measured IV curves did not show breakdown with the exception of one sensor, which showed evidence of micro-discharge at about 420V. This sensor was trained, after which no micro-discharge was found up to $1000 \mathrm{~V}$. The training consisted of keeping the sensor at the breakdown voltage (with the current limited to around $10 \mu \mathrm{A}$ ) for a few minutes until the current decreases. Estimations of the depletion voltage from capacitance measurements as a function of bias voltage gave depletion voltages between $199-245 \mathrm{~V}$ which satisfy the specifications $\left(\mathrm{V}_{\mathrm{fd}}<500 \mathrm{~V}\right)$.

Strip scan measurements were considered for studying the uniformity of electrical characteristics over the whole sensor. Coupling capacitance and bias resistance are very homogeneous for $99.95 \%$ of the strips and within specifications [17]. 
Coupling capacitances are between 66-68 pF/strip and bias resistance between 1.3-1.45 $\mathrm{M} \Omega$.

\section{Module Integration Concepts}

New designs are needed for the ATLAS tracker upgrade in terms of the module integration due to the requirements of sensor modularity. Such requirements demand important considerations in services (powering, cooling and readout) and important constraints in space and production time. The ATLAS Collaboration has proposed a straight stave for the barrel SCT and a petal stave for the end-caps [18].

A stave is a self contained object which integrates a number of sensor modules sharing the services of powering, cooling and readout. These are considered as a complete assembled unit instead of individual modules installed onto the barrel and end-cap structures. Staves may provide a double-sided (stereo) measurement with silicon covering either sides or an effective singled-sided measurement with partial coverage on any one side.

\subsection{The stave concept}

The straight stave consists of a core fabricated as a sandwich of carbon fiber facings around a spacing material such as honeycomb or foam. Within the spacing material is the cooling loop. On the facings an embedded kapton+copper bus cable distributes electrical services to each module. Sensors are glued to kapton hybrids and these glued to the bus tape. Fig.5 shows a recent prototype under construction to illustrate the details of this structure. It is $1.2 \mathrm{~m}$ long, with 48 segments per side and for $2.4 \mathrm{~cm}$ short strips. It is 10 chips wide (20 chips per hybrid). ABCN25 silicon strip readout chips have been used.

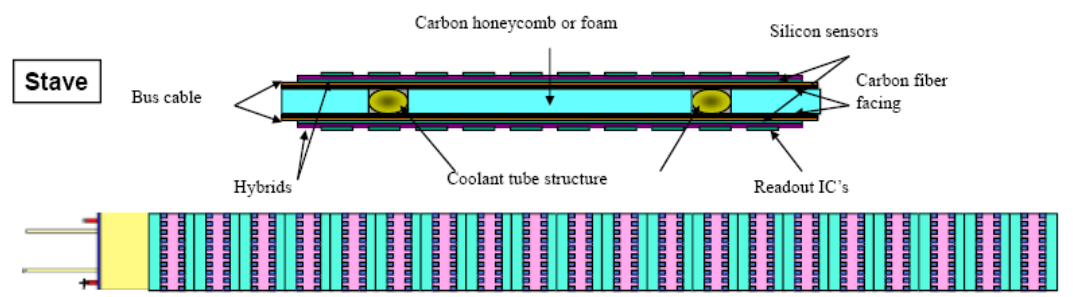

Fig. 5. Concept of an integrated barrel stave with short strip detectors. It is the prototype Stave-09 [18].

Earlier demonstrator prototypes were produced using ABCD3TA from the present ATLAS SCT together with serial powering lines [19]. The Stave-06 prototype was a six module stave with 4 chips wide hybrids. The p-in-n silicon sensors had $9.6 \mathrm{~cm}$ long strips. The results with this prototype were in agreement with the expected performance of the ABCD3TA chipset. No performance degradation was observed in the serial module chain when a module was unbiased. Another prototype with dimensions more representative of the ATLAS Tracker upgrade $(1 \mathrm{~m}$ length, 30 modules, 6 chips wide and detector with $3 \mathrm{~cm}$ strips) has been developed (Stave-07), using new hybrid and bus cable designs. It showed a good noise and data transmission performance. 


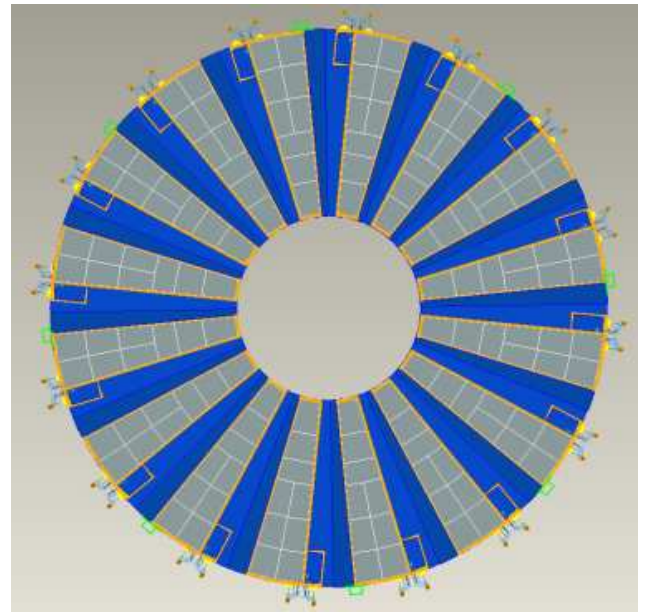

Fig. 6. End-cap disc simulation with 32 petal staves (grey)[20].

\subsection{The petal concept}

A stave with petal shape is the proposal for the end-cap of the ATLAS tracker upgrade. This structure follows quite closely the barrel stave concept. Each of the 5 endcap discs will be divided in 32 petals (16 on each side) as shown in Fig.6. Some of the challenges this structure implies are dealing with 6 different detector types mounted on petals depending on the inner radius, 9 different hybrids types and 116 chips per petal.

Thermal simulations [21] have been done to explore the behaviour at critical points such as are sensors and hybrids and avoid thermal runaway. Assuming a coolant temperature of $-30^{\circ} \mathrm{C}\left(-27^{\circ} \mathrm{C}\right.$ on the return pipe) the results show that the power consumption on sensors is within safety range $\left(2 \mathrm{~mW} / \mathrm{mm}^{2}\right)$ reaching a temperature around $-20^{\circ} \mathrm{C}$ that is adequate. It should be noted that wire-bonding angles will be demanding and it would imply the need of fan-ins to avoid possible shorts at chip edges.

\subsection{Alternative module design: Super-Module}

The baseline design for the tracker upgrade is based around staves/petals. Nevetheless, an alternative concept based on SCT experience with prototypes under development is studied. It is called Super-Module. It consists of individual double-sided modules with short strips sensors and bridged hybrids with ABCN ASICs inserted into a frame [22]. There are two proposals for super-module integration into cylinders:

1. Lateral Insertion. The integration of the super-modules is on the barrel cylinder and then all the structure is installed.

2. End-Insertion. The barrel structure can be assembled before the super-modules are integrated. The super-modules can be integrated by longitudinal mounting.

Thermal performance simulations show largely safe performance regarding thermal runaway.

\section{Summary}

The ATLAS Tracker will have to be replaced for the foreseen luminosity upgrade. Lots of R\&D is being pursued. ATLAS07 strip p-type sensors have been tested and they show very good electrical characteristics and performance being suitable for the short strip layers of the ATLAS tracker upgrade. 
Prototyping is vital and all the new features (such as hybrids, sensors, powering and cooling) have to be tested on realistic staves or petals. The stave community has built prototypes showing good electrical behaviour of the serial powering. A full prototype will be constructed in the coming months. The petal community is dealing with additional issues in the forward region, like integration of different types of hybrids, sensors, modules and large bonding angles.

\section{References}

[1] V. Baglin et al., Conceptual Design of the LHC Interaction Region Upgrade : Phase-I, CERNLHC-PROJECT-Report-1163

[2] L. Evans, SLHC accelerator and injector upgrades, SLHC-PP Kickoff Meeting, http://indico.cern.ch/conferenceOtherViews.py?view=standard\&confld=29254.

[3] R. Richter, Upgrade of ATLAS for High Luminosity at the Super-LHC, ATL-UPGRADE-PROC2009-001; ATL-COM-UPGRADE-2008-004- Geneva, CERN, 2008 - 3 p.

[4] A. Ahmad et al., The ATLAS Experiment at the CERN Large Hadron Collider, JINST 3 S08003, 2008.

[5] H.F.-W. Sadrozinski, Development of non-inverting Silicon strip detectors for the ATLAS ID upgrade. Available in: https://edms.cern.ch/file/816759/1/ATLAS_RD_SSD_May_18_06.doc.

[6] Summary of the current status of proposal. Available:http://atlas.web.cern.ch/Atlas/GROUPS/UPGRADES/

[7] Y. Unno, SLHC upgrade of the ATLAS SCT tracker, Nucl. Instr. and Meth. A (2009), doi:10.1016/j.nima.2009.08.008

[8] Hamamatsu Photonics, 325-6, Sunayama-cho, Hamamatsu City, Sizuoka Pref, 430-0193, Japan.

[9] Institutes: University of Liverpool, Jozef Stefan Institute (Ljubljana), University of California (Santa Cruz) and IFIC (Valencia), University of Tsukuba, and KEK.

[10] Cyclotron Radio Isotope Center (CYRIC), 6-3 Aramaki-Aoba, Aoba-ku, Sendai 980-8578, Japan.

[11] I. Mandic, http://www-f9.ijs.si/ mandic/Reactor.html.

[12] G. Lindstrom et al., Radiation hard silicon detectors - Developments by the RD48 (ROSE) collaboration. Nucl. Instr. And Meth. A 466 (2001), p. 308.

[13] A. Affolder, et al., Charge collection efficiencies of planar silicon detectors after reactor neutron and proton doses up to $1.6 \times 10^{16} \mathrm{n}_{\mathrm{eq}} \mathrm{cm}^{-2}$. Nucl. Instr. and Meth. A (2009), doi:10.1016/j.nima.2009.08.005.

[14] G. Kramberger, Performance of silicon pad detectors after mixed irradiations with neutrons and fast charged hadron, Nucl. Instr. and Meth. A, 609, (2009), 142-148.

[15] K. Hara, Testing of bulk radiation damage of n-in-p silicon sensor for very high radiation environment, $7^{\text {th }}$ International Hiroshima Symposium on the Development and Applications of Semiconductor Tracking Detectors, 29 August - 1 September, 2009.

[16] Institutes: University of Cambridge, Stony Brook University, Charles University (Prague), and The University of Geneva. 
[17] ATLAS SCT Collaboration, Supply of Silicon Microstrip Sensors of ATLAS07 Specification. V 4.5 2007. https://edms.cern.ch/file/888375/1/ATLAS07TechnicalSpecsR4.5.pdf.

[18] J. Kierstad, et al., Development of large area integrated silicon tracking elements for the LHC luminosity upgrade, Nucl. Instr. And Meth. A 579 (2007) 801.

[19] P.W. Phillips et al., Serial Powering of silicon strip modules for the ATLAS tracker upgrade, Topical Workshop on Electronics for Particle Physics, Naxos, Greece, 15 - 19 Sep 2008, pp. 309

[20] Image provided by David Santoyo (david.santoyo@ific.uv.es),IFIC, Valencia.

[21] D. Santoyo, Forward Region Concepts; Petal Concept \& FEA, SLHC Stave/Petal Upgrade Mechanics Workshop, 13-15, January, 2009, LBNL (USA).

[22] S. González-Sevilla, A silicon strip-module for the ATLAS Inner Detector upgrade in the sLHC collider, $7^{\text {th }}$ International Hiroshima Symposium on the Development and Applications of Semiconductor Tracking Detectors, 29 August - 1 September, 2009. 